\title{
ANALYSIS OF BROADBAND ANTENNA RADIATION PULSES
}

\author{
Aleksander Cherenkov $^{\mathrm{a}^{*}}$, Taras Hutsol ${ }^{\mathrm{b}}$, Igor Garasymchuk ${ }^{\mathrm{b}}$, Jurii Pancyr ${ }^{\mathrm{b}}$, \\ Dmytro Terenov ${ }^{\mathrm{b}}$, Vitalii Dubyna ${ }^{\mathrm{b}}$ \\ ${ }^{a}$ Educational and Scientific Institute of Power Energy and Computer Technologies, State Technical \\ University in Charków \\ ${ }^{\mathrm{b}}$ Faculty of Engineering and Technology, State Agrarian and Engineering University in Podilia ${ }^{\mathrm{b}}$ \\ Department of Environmental Protection, Warsaw University of Life Sciences
}

"Corresponding author: e-mail: pro-gp@pdatu.edu.ua

\begin{tabular}{l}
\hline ARTICLE INFO \\
\hline Article history: \\
Received: September 2017 \\
Received in the revised form: \\
November 2017 \\
Accepted: Junuary 2018 \\
\hline Key words: \\
antenna, \\
electromagnetic energy emitter, \\
Fourier transform, \\
communicating wave, \\
aperture, \\
biotechnology, \\
broadband pulses
\end{tabular}

\section{ABSTRACT}

Electromagnetic energy can alter metabolic and biosynthetic processes
and under certain parameters of pulsed EMF it can change pulse
repetition frequency, operation cycle, power, exposure, as well as it
can slow down and inhibit cell growth. MW irradiation range of RNA
and DNA - containing virus reduces their infectivity. Inhibition of
bacterial cultures growth, changes in phagocytic activity of protein
biosynthesis, ultrastructural changes in the cells when exposed to
EMF EHF. It was found in experiments with micro-organisms that
biological effects of EMF on microorganisms wore a resonant charac-
ter. One of the basic mechanisms of inhibitory action of EHF radiation
on harmful microorganisms is the role of membranes in biological
reactions of microorganisms on the EMR.

\section{Постановка проблемы}

Мощные импульсы широкополосного излучения находят применение в радиолокации удаленных объектов, исследовании электромагнитной (ЭМ) совместимости и устойчивости радиоэлектронных систем в условиях воздействия сильных электромагнитных полей (ЭМП). Кроме того, они могут быть с успехом использованы для борьбы с вредителями, обитающими в подповерхностном слое почвы. Каждое конкретное применение накладывает свои требования на параметры широкополосных (ШП) импульсов и излучающую систему. Так использование ШП импульсов пикосекундной длительности для радиолокации объектов, удаленных на большие расстояния ( 100 км), является невозможным. Это связано с дисперсией атмосферы, которая приводит к расплыванию импульса при его распространении уже на расстояниях $<5$ км.

Аналогичная ситуация имеет место в исследованиях по электромагнитной совместимости и устойчивости радиоэлектронных систем. Как оказалось, пикосекундные импульсы не оказывают существенного влияния на функционирование различных систем, вследствие конечного времени их реакции на 
воздействие импульса. Поэтому для этих исследований используются ШП импульсы наносекундной длительности (5-10 нс).

В случае борьбы с вредителями также необходимы ШП импульсы наносекундной длительности. Поскольку пикосекундные импульсы не приведут к необратимым процессам в клетках вредителей.

\section{Анализ последних исследований и публикаций}

Для излучения ШП импульсов применяются различные антенные системы. Можно выделить, в основном, два типа антенн: антенны с параболическим рефлектором, получившие название IRA (Impulse Radiating Antenna (Gubanov 1994; Baum 1993)) и ТЕМ антенны (Nowak, Gucol, Bendera, 2006, Giri 1997). Для наших целей антенны IRA не подходят по причине больших размеров. Обычно их апертура может достигать 4 м. ТЕМ антенна, как правило, выполнена из двух треугольных пластин различного профиля, обеспечивающих плавный переход от волнового сопротивления генератора к волновому сопротивлению свободного пространства.

Такая антенна является антенной бегущей волны, поэтому она имеет длину, соответствующую длине волны нижней границы спектра излучаемого импульса. В качестве фидера обычно используются полосковые или коаксиальные линии. Выполненные к настоящему времени исследования (Hala 2010, Kalinichev, 2009, Kun, 1967) показывают, что основной недостаток таких антенных систем - это их большая длина по сравнению с пространственной длительностью возбуждающего импульса.

Таким образом, на основании всего сказанного, можно сделать вывод о том, что наиболее часто используемые антенные системы для передачи ШП импульсов для наших конкретных целей не подходят из-за их значительных геометрических размеров.

\section{Постановка задачи}

Создание энергосберегающий, экологически чистой и эффективной радиоимпульсной ЭМ биотехнологии, а также электронной системы импульсной электромагнитной энергии. Теоретическое обоснование по созданию импульсного источника электромагнитной энергии и широкополосной антенны излучения.

\section{Изложение основного материала}

Представим излучающую поверхность плоской и прямоугольной. Пространственная геометрия задачи показана на рис. 1. Для получения конкретных результатов будем рассматривать случай возбуждения апертуры плоской равноамплитудной синфазной волной. 


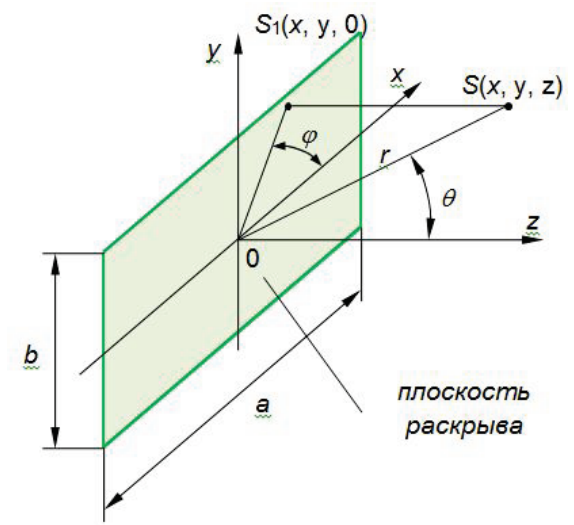

Рисунок 1. Геометрия задачи

Выпишем поля излучения в дальней зоне, которые в сферической системе координат при $r \rightarrow \infty$ имеют вид:

$$
\begin{aligned}
& E_{\theta}(\omega, r, \theta, \varphi)=\frac{i k}{4 \pi} \cos \varphi(1+\cos \theta) \frac{e^{-i k r}}{r} F(\theta, \varphi) \\
& E_{\varphi}(\omega, r, \theta, \varphi)=\frac{i k}{4 \pi} \sin \varphi(1+\cos \theta) \frac{e^{-i k r}}{r} F(\theta, \varphi)
\end{aligned}
$$

где:

$k=\omega \sqrt{\varepsilon_{0} \mu_{0}}, k_{y}=k \sin \theta \sin \varphi$

$$
F(q, f)={\underset{-a / 2}{a / 2}}_{-b / 2}^{b / 2} E_{y}(x, y) e^{i\left(k_{x} x+k_{y} y\right)} d x d y .
$$

Угол $\theta$ отсчитывается от оси $z$, а угол $\varphi$ - от оси $x$ против часовой стрелки.

$E_{y}(x, y)$ - преобразование Фурье возбуждающего поля в растре рупорной антенны, причем:

$$
E_{y}(x, y)=E_{0} F(\omega)
$$

где:

$E_{0}$ - амплитудный коэффициент,

$F(\omega)$ - спектр возбуждающего сигнала.

Рассмотрим выражение (3), которое, после подстановки в него (4), можем записать в аналитическом виде: 


$$
\begin{aligned}
F(\theta, \varphi) & =E_{0} F(\omega)=\frac{\left(e^{i k_{x} a / 2}-e^{-i k_{x} a / 2}\right)\left(e^{i k_{y} b / 2}-e^{-i k_{y} b / 2}\right)}{i k_{x} i k_{y}}= \\
& =4 E_{0} F(\omega) \frac{\sin \left(\frac{k_{x} a}{2}\right) \sin \left(\frac{k_{y} b}{2}\right)}{k_{x} k_{y}}
\end{aligned}
$$

Для дальнейшего рассмотрения введем функцию:

$$
\Phi(x)=\sin x / x
$$

тогда соотношение (5) примет вид:

$$
F(\theta, \varphi)=E_{0} F(\omega) a b \Phi\left(\frac{k_{x} a}{2}\right) \Phi\left(\frac{k_{y} b}{2}\right)
$$

Подставляя (6) в выражение (1), получим уравнение для $E_{\theta}$ компоненты поля:

$$
\begin{aligned}
E_{\varphi}(\omega, r, \theta, \varphi)=\frac{i k}{4 \pi} \cos \varphi(1 & +\cos \theta) \frac{e^{-i k r}}{r} E_{0} F(\omega) a b \Phi\left(\frac{k_{x} a}{2}\right) \times \\
& \times \Phi\left(\frac{k_{y} b}{2}\right)
\end{aligned}
$$

По аналогии запишем соотношение для $E_{\varphi}$ компоненты поля. После подстановки (6) в (3) получим:

$$
\begin{aligned}
E_{\varphi}(\omega, r, \theta, \varphi)=\frac{i k}{4 \pi} \sin \varphi( & 1+\cos \theta) \frac{e^{-i k r}}{r} E_{0} F(\omega) a b \Phi\left(\frac{k_{x} a}{2}\right) \times \\
& \times \Phi\left(\frac{k_{y} b}{2}\right)
\end{aligned}
$$

Для перехода во временную область по $E_{\theta}$ и $E_{\varphi}$ компонентам используем обратное преобразование Фурье (Giri, 1997):

$$
\begin{gathered}
E_{\theta}(t, r, \theta, \varphi)=\frac{1}{2 \pi} \int_{-\infty}^{\infty} E_{\theta}(\omega, r, \theta, \varphi) e^{-i \omega t} d \omega \\
E_{\varphi}(t, r, \theta, \varphi)=\frac{1}{2 \pi} \int_{-\infty}^{\infty} E_{\varphi}(\omega, r, \theta, \varphi) e^{+i \omega t} d \omega
\end{gathered}
$$

При произвольных координатах уравнения (9) и (10) в конечном виде не разрешимы. Поэтому, интегрирование необходимо вести численными методами. 
Analysis of broadband...

Теперь определим характеристику направленности рупорного излучателя в дальней зоне в двух взаимно перпендикулярных плоскостях по энергетическому показателю излучения в каждом из направлений:

$$
\begin{gathered}
G_{1}(\theta, \varphi=0)=\frac{\int_{-\infty}^{\infty}\left|E_{\theta}(t, r, \theta, 0)\right|^{2} d t}{\int_{-\infty}^{\infty}\left|E_{\theta}(t, r, 0,0)\right|^{2} d t}, \\
G_{2}\left(\theta, \varphi=\frac{\pi}{2}\right)=\frac{\int_{-\infty}^{\infty}\left|E_{\varphi}\left(t, r, \theta, \frac{\pi}{2}\right)\right|^{2} d t}{\int_{-\infty}^{\infty}\left|E_{\varphi}\left(t, r, 0, \frac{\pi}{2}\right)\right|^{2} d t .}
\end{gathered}
$$

Величины и $G_{2}\left(\theta, \varphi=\frac{\pi}{2}\right)$ представляют собой энергетические диаграммы направленности (ЭДН).

Для того, чтобы построить сечения $G_{1}(\theta, \varphi=0)$ и $G_{2}\left(\theta, \varphi=\frac{\pi}{2}\right)$ при различных параметрах антенны, необходимо учесть следующее. Согласно теореме Парсеваля:

$$
\int_{-\infty}^{\infty}\left|E_{\theta}(t, r, \theta, \varphi)\right|^{2} d t=\int_{-\infty}^{\infty}\left|E_{\theta}(\omega, r, \theta, \varphi)\right|^{2} d \omega
$$

и аналогично:

$$
\int_{-\infty}^{\infty}\left|E_{\varphi}(t, r, \theta, \varphi)\right|^{2} d t=\int_{-\infty}^{\infty}\left|E_{\varphi}(\omega, r, \theta, \varphi)\right|^{2} d \omega
$$

Эти соотношения, в сущности, представляют собой формулировку закона сохранения энергии в двух областях (временной и частотной). С учетом (13) и (14) выражения (11), (12) примут вид:

$$
\begin{gathered}
G_{1}(\theta, \varphi=0)=\frac{\int_{-\infty}^{\infty}\left|E_{\theta}(\omega, r, \theta, 0)\right|^{2} d \omega}{\int_{-\infty}^{\infty}\left|E_{\theta}(\omega, r, 0,0)\right|^{2} d \omega}, \\
G_{2}\left(\theta, \varphi=\frac{\pi}{2}\right)=\frac{\int_{-\infty}^{\infty}\left|E_{\varphi}\left(\omega, r, \theta, \frac{\pi}{2}\right)\right|^{2} d \omega}{\int_{-\infty}^{\infty}\left|E_{\varphi}\left(\omega, r, 0, \frac{\pi}{2}\right)\right|^{2} d \omega} .
\end{gathered}
$$

Сначала рассмотрим интеграл, стоящий в знаменателе уравнения (15). После подстановки в него значения $E_{\theta}(\omega, r, 0,0)$, можем записать: 


$$
\int_{-\infty}^{\infty}\left|E_{\theta}(\omega, r, 0,0)\right|^{2} d \omega=\frac{E_{0}^{2} \varepsilon_{0} \mu_{0} a^{2} b^{2}}{4 \pi^{2} r^{2}} \int_{-\infty}^{\infty} \omega^{2}|F(\omega)|^{2} d \omega
$$

При этом интеграл в числителе (15) преобразуется к виду:

$$
\begin{gathered}
\int_{-\infty}^{\infty}\left|E_{\theta}(\omega, r, \theta, 0)\right|^{2} d \omega=\frac{E_{0}^{2} \varepsilon_{0} \mu_{0}(1+\cos \theta)^{2} a^{2} b^{2}}{16 \pi^{2} r^{2}} \times \\
\times \int_{-\infty}^{\infty} \omega^{2}|F(\omega)|^{2} \Phi^{2}\left(\frac{k_{x} a}{2}\right) d \omega
\end{gathered}
$$

Здесь мы учитываем, что при $\varphi \rightarrow 0 \quad \Phi\left(k_{y} b / 2\right)=1$. Рассмотрим $\Phi^{2}\left(k_{x} a / 2\right)$. Как было показано выше, в общем случае $k_{x}=k \sin \theta \cos \varphi$. Тогда с учетом принятых обозначений можем записать:

$$
\Phi^{2}\left(\frac{k_{x} a}{2}\right)=\left[\frac{\sin \left(\frac{k a}{2} \sin \theta\right)}{\frac{k a}{2} \sin \theta}\right]^{2}=\left[\frac{\sin \left(\frac{\omega \sqrt{\varepsilon_{0} \mu_{0}} a}{2} \sin \theta\right)}{\frac{\omega \sqrt{\varepsilon_{0} \mu_{0}} a}{2} \sin \theta}\right]^{2} .
$$

Теперь запишем в окончательном виде с учетом (17), (18) выражение (15), которое определяет Эдн пирамидального рупора в дальней зоне в плоскости $x 0 z$ (см. рис. 1):

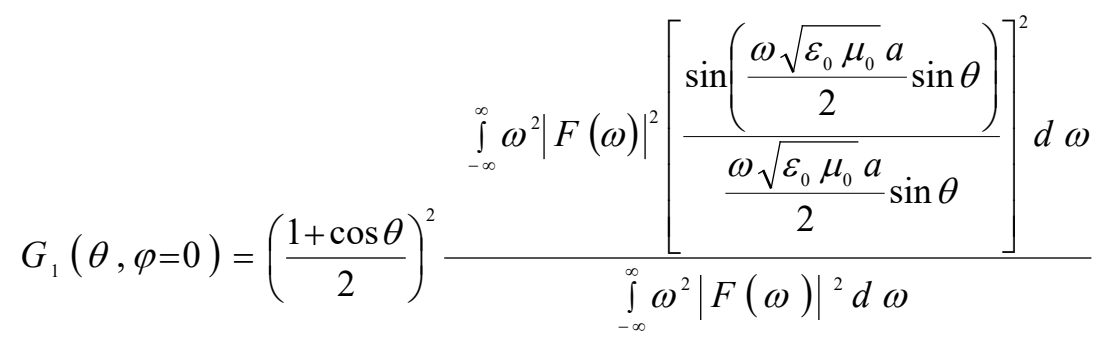

По аналогии, как мы только что сделали, проанализируем соотношение (16). Его знаменатель можем представить в виде:

$$
\int_{-\infty}^{\infty}\left|E_{\varphi}\left(\omega, r, 0, \frac{\pi}{2}\right)\right|^{2} d \omega=\frac{E_{0}^{2} \varepsilon_{0} \mu_{0} a^{2} b^{2}}{4 \pi^{2} r^{2}} \int_{-\infty}^{\infty} \omega^{2}|F(\omega)|^{2} d \omega
$$

Числитель уравнения (16) при $\varphi=\pi / 2$ примет вид: 
Analysis of broadband...

$$
\begin{gathered}
\int_{-\infty}^{\infty}\left|E_{\varphi}\left(\omega, r, \theta, \frac{\pi}{2}\right)\right|^{2} d \omega=\frac{E_{0}^{2} \varepsilon_{0} \mu_{0} a^{2} b^{2}}{16 \pi^{2} r^{2}}(1+\cos \theta)^{2} \times \\
\times \int_{-\infty}^{\infty} \omega^{2}|F(\omega)|^{2} \Phi^{2}\left(\frac{k_{y} b}{2}\right) d \omega
\end{gathered}
$$

Рассмотрим соотношение $\Phi^{2}\left(k_{y} a / 2\right)$, которое с учетом того, что $k_{y}=k \sin \theta \sin \varphi$, можем записать в следующем виде:

$$
\Phi^{2}\left(\frac{k_{y} b}{2}\right)=\left[\frac{\sin \left(\frac{k b}{2} \sin \theta\right)}{\frac{k b}{2} \sin \theta}\right]^{2}=\left[\frac{\sin \left(\frac{\omega \sqrt{\varepsilon_{0} \mu_{0}} b}{2} \sin \theta\right)}{\frac{\omega \sqrt{\varepsilon_{0} \mu_{0}} b}{2} \sin \theta}\right]^{2} .
$$

Теперь запишем в окончательном виде выражение (16) с учетом соотношений (21)...(23):

$$
G_{2}\left(\theta, \varphi=\frac{\pi}{2}\right)=\left(\frac{1+\cos \theta}{2}\right)^{2} \frac{\int_{-\infty}^{\infty} \omega^{2}|F(\omega)|^{2}\left[\frac{\sin \left(\frac{\omega \sqrt{\varepsilon_{0} \mu_{0}} b}{2} \sin \theta\right)}{\frac{\omega \sqrt{\varepsilon_{0} \mu_{0}} b}{2} \sin \theta}\right]^{2} d \omega}{\int_{-\infty}^{\infty} \omega^{2}|F(\omega)|^{2} d \omega}
$$

Это соотношение определяет ЭДН пирамидального рупора в дальней зоне в плоскости $y 0 z$.

После того, как мы получили соотношения (20) и (24), определяющие ЭДН системы пирамидальный рупорно-широкополосный сигнал в двух взаимноперпендикулярных плоскостях, определим пространственно-временные характеристики поля. Считаем, что на вход прямоугольного волновода сечением $23 \times 10$ мм подается сигнал $s(t)$, представляющий собой гармонические колебания на частоте $f_{u}=10$ ГГц, модулированные по амплитуде гауссовым импульсом длительностью $\beta=100$ нс (см. рис. 2):

$$
s(t)=A t e^{-\left(\frac{t}{\beta}\right)^{2}} \cos ^{2}\left(2 \pi f_{H} t\right)
$$




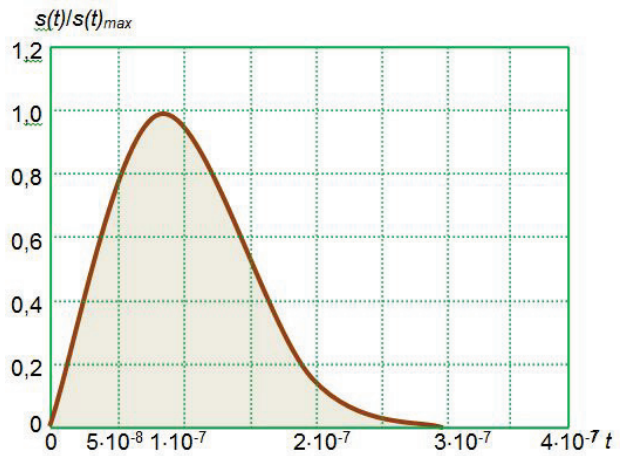

Рис. 2. Сигнал на входе антенны

Спектр такого сигнала найдем, используя преобразование Фурье:

$$
F(\omega)=\frac{1}{2 \pi} \int_{-\infty}^{\infty} s(t) e^{-i \omega t} d t
$$

В качестве первого шага рассмотрим сигнал, представляющий собой видеоимпульс:

$$
s_{1}(t)=A t e^{-\left(\frac{t}{\beta}\right)^{2}}
$$

тогда спектр такого сигнала определим из выражения

$$
F_{1}(\omega)=\frac{1}{2 \pi} \int_{-\infty}^{\infty} S_{1}(t) e^{-i \omega t} d t=\frac{A}{2 \pi} \int_{-\infty}^{\infty} t e^{-\frac{1}{\beta^{2}} t^{2}-i \omega t} d t
$$

С учетом табличного интеграла (Kozak, Gordyjchuk, Semenyshyna, Vylchynskaya, 2015):

$$
\int_{-\infty}^{\infty} x^{n} e^{-p x^{2}+2 q x} d x=\frac{1}{2^{n-1} p} \sqrt{\frac{\pi}{p}} \frac{d^{n-1}}{d q^{n-1}}\left(q e^{\frac{q^{2}}{p}}\right) ; p>0
$$

Запишем соотношение (28) в окончательном виде:

$$
F_{1}(\omega)=-\frac{i A \omega \beta^{3}}{4 \sqrt{\pi}} e^{-\frac{\omega^{2} \beta^{2}}{4}}
$$

Теперь найдем спектр нашего радиоимпульса. После подстановки уравнения (25) в выражение (26), с учетом электронной мощности однодиодного генератора, получим:

$$
F(\omega)=\frac{1}{2 \pi} \int_{-\infty}^{\infty} s_{1}(t)\left(\cos \omega_{H} t\right)^{2} d t
$$


Analysis of broadband...

Если теперь преобразуем тригонометрическую функцию $\left(\cos \omega_{н} t\right)^{2}=\left(1+\cos 2 \omega_{н} t\right) / 2$, то соотношение (30) примет вид:

$$
F(\omega)=\frac{1}{2 \pi} \frac{1}{2}\left[\int_{-\infty}^{\infty} S_{1}(t) e^{-i \omega t} d t+\int_{-\infty}^{\infty} S_{1}(t) \cos 2 \omega_{H} t e^{-i \omega t} d t\right]
$$

Рассмотрим первый интеграл в (31), который с учетом (29) запишем в виде:

$$
F_{2}(\omega)=\frac{1}{2} \frac{1}{2 \pi} \int_{-\infty}^{\infty} S_{1}(t) e^{-i \omega t} d t=\frac{F_{1}(\omega)}{2}=-\frac{i A \omega \beta^{3}}{8 \sqrt{\pi}} e^{-\frac{\omega^{2} \beta^{2}}{4}}
$$

Рассмотрим второй интеграл в выражении (31):

$$
F_{3}(\omega)=\frac{1}{2} \frac{1}{2 \pi} \int_{-\infty}^{\infty} S_{1}(t) \cos 2 \omega_{H} t e^{-i \omega t} d t
$$

Представим тригонометрическую функцию в виде показательно:

$$
\cos 2 \omega_{n} t=\frac{e^{i 2 \omega_{n} t}+e^{-i 2 \omega_{n} t}}{2}
$$

тогда выражение (33) преобразуется к виду:

$$
F_{3}(\omega)=\frac{1}{4} \frac{1}{2 \pi}\left[\int_{-\infty}^{\infty} S_{1}(t) e^{-i\left(\omega-2 \omega_{\mathrm{H}}\right) t} d t+\int_{-\infty}^{\infty} S_{1}(t) e^{-i\left(\omega+2 \omega_{\mathrm{H}}\right) t} d t\right]
$$

Рассмотрим интеграл:

$$
F_{3}^{\prime}(\omega)=\frac{1}{4} \frac{1}{2 \pi} \int_{-\infty}^{\infty} S_{1}(t) e^{-i\left(\omega-2 \omega_{\mathrm{H}}\right) t} d t
$$

Если сравнить полученное выражение с соотношениями (28) и (29), то можем записать в окончательном виде:

$$
F_{3}^{\prime}(\omega)=\frac{F_{1}\left(\omega-2 \omega_{\mathrm{H}}\right)}{4}=-\frac{i A\left(\omega-2 \omega_{\mathrm{H}}\right) \beta^{3}}{16 \sqrt{\pi}} e^{-\frac{\left(\omega-2 \omega_{\mathrm{H}}\right)^{2} \beta^{2}}{4}}
$$

В качестве следующего шага проанализируем интеграл:

$$
F_{3}^{\prime \prime}(\omega)=\frac{1}{4} \frac{1}{2 \pi} \int_{-\infty}^{\infty} S_{1}(t) e^{-i\left(\omega+2 \omega_{\mathrm{H}}\right) t} d t
$$

и по аналогии с предыдущим интегралом сразу запишем:

$$
F_{3}^{\prime \prime}(\omega)=\frac{F_{1}\left(\omega+2 \omega_{\mathrm{H}}\right)}{4}=-\frac{i A\left(\omega+2 \omega_{\mathrm{H}}\right) \beta^{3}}{16 \sqrt{\pi}} e^{-\frac{\left(\omega+2 \omega_{\mathrm{H}}\right)^{2} \beta^{2}}{4}}
$$

С учетом выражений (34), (36) и (38) представим спектр нашего радиоимпульса, который первоначально определялся соотношением (32): 


$$
\begin{aligned}
F(\omega)=F_{2}(\omega)+F_{3}^{\prime}(\omega)+ & F_{3}^{\prime \prime}(\omega)=-\frac{i A \beta^{3}}{8 \sqrt{\pi}}\left[\omega e^{-\frac{\omega^{2} \beta^{2}}{4}}+\frac{\left(\omega-2 \omega_{n}\right)}{2} e^{-\frac{\left(\omega-2 \omega_{n}\right)^{2} \beta^{2}}{4}}+\right. \\
+ & \left.\frac{\left(\omega+2 \omega_{n}\right)}{2} e^{-\frac{\left(\omega+2 \omega_{n}\right)^{2} \beta^{2}}{4}}\right]
\end{aligned}
$$

Теперь, зная $F(\omega)$, запишем в окончательном виде выражение (20), которое определяет ЭДН пирамидального рупора в дальней зоне в плоскости $x 0 z$ (см. рис. 1):

$$
\begin{gathered}
G_{1}(\theta, \varphi=0)=\left(\frac{1+\cos \theta}{2}\right)^{2} \frac{\int_{-\infty}^{\infty} \omega^{2}\left[\omega e^{-\frac{\omega^{2} \beta^{2}}{4}}+\frac{\left(\omega-2 \omega_{n}\right)}{2} e^{-\frac{\left(\omega-2 \omega_{n}\right)^{2} \beta^{2}}{4}}+\int_{-\infty}^{2}\left[\omega e^{-\frac{\omega^{2} \beta^{2}}{4}}+\frac{\left(\omega-2 \omega_{n}\right)}{2} e^{-\frac{\left(\omega-2 \omega_{n}\right)^{2} \beta^{2}}{4}}+\right.\right.}{\left.+\frac{\left(\omega+2 \omega_{n}\right)}{2} e^{-\frac{\left(\omega+2 \omega_{n}\right)^{2} \beta^{2}}{4}}\right]\left[\frac{\sin \left(\frac{\omega \sqrt{\varepsilon_{0} \mu_{0}} a}{2} \sin \theta\right)}{\frac{\omega \sqrt{\varepsilon_{0} \mu_{0}} a}{2} \sin \theta}\right] d \omega} \\
\left.+\frac{\left(\omega+2 \omega_{n}\right)}{2} e^{-\frac{\left(\omega+2 \omega_{n}\right)^{2} \beta^{2}}{4}}\right]^{2} d \omega
\end{gathered}
$$

Аналогичным образом, используя выражения (24) и (39), запишем соотношение, определяющее ЭДН пирамидального рупора в дальней зоне в плоскости $y 0 z$ (см. рис. 1).

$$
\begin{gathered}
G_{2}\left(\theta, \varphi=\frac{\pi}{2}\right)=\left(\frac{1+\cos \theta}{2}\right)^{2} \frac{\int_{-\infty}^{\infty} \omega^{2}\left[\omega e^{-\frac{\omega^{2} \beta^{2}}{4}}+\frac{\left(\omega-2 \omega_{n}\right)}{2} e^{-\frac{\left(\omega-2 \omega_{n}\right)^{2} \beta^{2}}{4}}+\right.}{\int_{-\infty}^{\infty} \omega^{2}\left[\omega e^{-\frac{\omega^{2} \beta^{2}}{4}}+\frac{\left(\omega-2 \omega_{H}\right)}{2} e^{-\frac{\left(\omega-2 \omega_{n}\right)^{2} \beta^{2}}{4}}+\right.} \\
+\frac{\left.\frac{\left(\omega+2 \omega_{u}\right)}{2} e^{-\frac{\left(\omega+2 \omega_{u}\right)^{2} \beta^{2}}{4}}\right]^{2}\left[\frac{\sin \left(\frac{\omega \sqrt{\varepsilon_{0} \mu_{0}} b}{2} \sin \theta\right)}{\frac{\omega \sqrt{\varepsilon_{0} \mu_{0}} b}{2} \sin \theta}\right] d \omega}{\left.+\frac{\left(\omega+2 \omega_{u}\right)}{2} e^{-\frac{\left(\omega+2 \omega_{u}\right)^{2} \beta^{2}}{4}}\right]^{2} d \omega}
\end{gathered}
$$


Analysis of broadband...

Если сравнить выражения (40) и (41), то нетрудно заметить, что для получения аксиально-симметричной эдн раскрыв рупора должен быть квадратным. Этот вывод хорошо согласуется с результатами работы (Chernyshev, 2001).

Поэтому расчет эдн системы пирамидальный рупор-сигнал проведем для плоскости $x 0 z$. Для этого используем выражение (40).

Все расчеты будем проводить для дальней зоны (зоны Фраунгофера), которая определяется как (Chernyshev, 2001):

$$
r=10 \frac{f_{n} D^{2}}{V_{\phi}},
$$

где:

$D$ - максимальный габарит апертуры, который в нашем случае равен раскрыву рупора,

$V_{\phi}$ - фазовая скорость эм волны в бреде распространения, которая для рассматриваемого случая равна скорости света $C$.

Для размеров раскрыва пирамидального рупора $10 \ldots 20$ см дальняя зона составляет примерно $1 \mathrm{M}$.

Результаты расчета разрезов $G_{1}(\theta, \varphi=0)$ представлены на рис. 3 для трех размеров раскрыва пирамидального рупора (кривая $1, a=10 \mathrm{cm;} \mathrm{кривая} 2, a=15$ см; кривая 3, $a=20$ см). Как видно из рисунка для широкополосных сигналов эдн более гладкие, чем классические монохроматические диаграммы направленности для того же пирамидального рупора. Это хорошо согласуется с результатами работ [Ymmoreev, 2001; Terenov and Gutsol, 2015). Также необходимо отметить, что чем больше раскрыв рупора, тем уже диаграмма направленности (см. рис. 3).

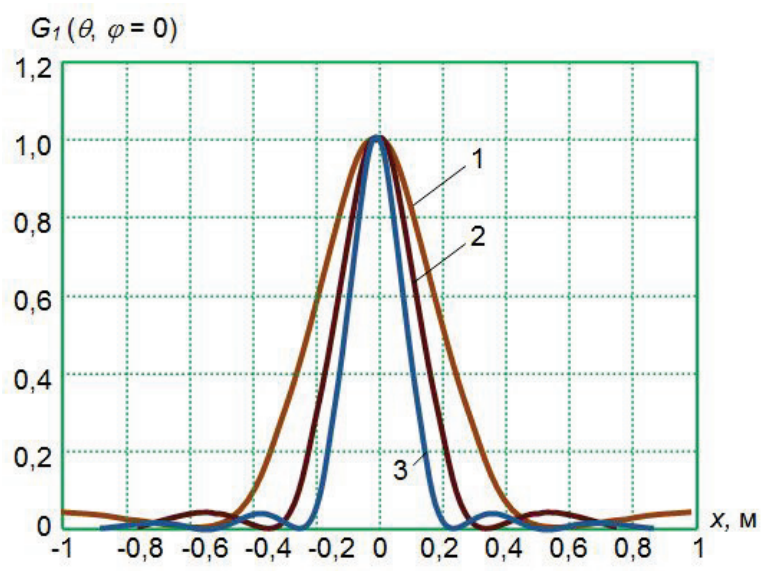

Рисунок 3. Разрезы эдН системы пирамидальный рупор-сигнал для различных размеров раскрыва 
Однако в случае узких эдН будет иметь место "рассыпание" сигнала при отклонении от направления $\theta=\varphi=0$. С другой стороны, для эффективной борьбы с вредителями в почве желательно иметь более широкую диаграмму направленности. Именно поэтому мы ограничиваемся размерами раскрыва рупора $a \times b=10 \times 10 \mathrm{~cm}$. В этом случае ширина диаграммы направленности по уровню $G_{1}(\theta, \varphi=0) / 2$ составляет порядка 50 см.

Для определения длины рупорного излучателя, возбуждаемого импульсным сигналом, воспользуемся результатами работы (Ymmoreev, 2010). Это связано с тем, что в случае негармонических сигналов, также как и гармонических, распределение поля в раскрыве излучателя должно быть синфазным (Voskresensky, 1972). Хорошо известно, что максимальная фазовая ошибка в раскрыве $\psi_{\max }$ определяется геометрическими размерами рупора. При этом ее допустимая величина должна удовлетворять следующим условиям (Voskresensky, 1972):

- в $H$-плоскости:

$$
\psi_{\max }=\frac{\pi a^{2}}{4 \lambda_{H} R_{H}} \leq \frac{3}{4} \pi
$$

- в $E$-плоскости:

$$
\psi_{\max }=\frac{\pi b^{2}}{4 \lambda_{H} R_{E}} \leq \frac{\pi}{2}
$$

где:

$R_{H} \quad-$ расстояние от раскрыва до точки, в которой сходятся ребра рупора в плоскости вектора $E$ основной волны в волноводе;

$R_{E} \quad-$ расстояние от раскрыва до точки, в которой сходятся ребра рупора в плоскости вектора $H$ волны $T E_{10}$ в волноводе;

$\lambda_{\mu}=3 \mathrm{~cm}$

В итоге, из выражений (43) и (44) легко получить соотношения, которые определяют длину рупора в плоскостях $H$ и $E$ :

$$
\begin{aligned}
& R_{H} \geq\left(a^{2} / 3 \lambda_{H}\right) \\
& R_{E} \geq\left(b^{2} / 2 \lambda_{H}\right)
\end{aligned}
$$

При этом, с другой стороны, размеры $R_{H}$ и $R_{E}$ связаны с размерами раскрыва $a, b$ и с размерами волновода $a_{6}, b_{\text {в }}$ соотношением:

$$
\frac{R_{H}}{R_{E}}=\frac{a}{b} \frac{\left(b-b_{\theta}\right)}{\left(a-a_{\theta}\right)} .
$$


Analysis of broadband...

Методика определения длины рупорного излучателя состоит в следующем. В выражении (45) знак неравенства заменяем на равенство и находим $R_{H}$. После этого по формуле (47) определяем $R_{E}$, учитывая, что стандартное сечение прямоугольного волновода трехсантиметрового диапазона длина волн $a_{b} \times b_{s}=23 \times 10$ мм. Полученное таким образом значение должно удовлетворять неравенству (46). После подстановки численных значений найдем, что для получения синфазного распределения поля в раскрыве длина рупорного излучателя от раскрыва до фазового фокуса должна быть равна 17 см. При этом длина рупора от раскрыва до соединения с волноводом составляет $13 \mathrm{~cm}$.

\section{Выводы}

1. Для уничтожения насекомых вредителей в почве импульсным электромагнитным излучением необходимо использовать широкополосный пирамидальный рупорный излучатель с параметрами: $a \times b=10$ см х 10 см; ширина диаграммы направленности более 50 см, длина рупора 130 мм.

2. Получила дальнейшее развитие теория анализа широкополосных антенн, которая отличается от известных тем, что в ней обоснованы параметры для излучения радиоимпульсов сантиметрового диапазона с малой величиной длительности импульсов.

\section{Библиографический список}

Gubanov, V. P. (1994). Generation of powerful nanosecond pulses of electromagnetic radiation / Gubanov V. P. [and etc.]. Letters to the Journal of Technical Physics, T. 20, № 14. p. 89-93.

Baum, C. E. (1993). Impulse radiating antennas. Baum C. E., Farr E. G. Ultra-Wideband, Short-Pulse Electromagnetics. New York, Plenum Press, p. 139-147.

Nowak, J., Gucol, T., Bendera, I. (2006). Mechaniczne niszczenie stonki ziemniaczanej, Ziemniak Polski, 4, 30-33.

Giri, D.V. (1997). Desigh, Fabrication, and Testing of a Paraboloidal Reflector Antenna and Pulser System for Impulse-Like Waveforms. Giri D. V., Lackner H., Smith I. D., Morton D. W., Baum C. E., Marek J. R., Prather W. D., and Scholfield D. W. IEEE Trans. Plasma Sci. Vol. 25, p. 318326.

Hala, A.V. (2010). The algorithm for calculating the ultrawideband antenna / Hala A. V., Korolkov A. V. Mathematical morphology. Electron. Matemathematical and Medico-biological Journal. T. 9, Issue. 1. URL: http://www.smolensk.ru.

Kozak, A., Gordyjchuk, Y., Semenyshyna, Y., Vylchynskaya, D. (2015). Analysis of the process of the action of electromagnetic impulse signals on biological pests in the soil. MOTROL. Commission of motorization and energetics in agriculture: Polish Academy of sciences. Vol. 17, №1. 4550 .

Chernyshev, S.L. (2001). Spatio-temporal analysis of the characteristics of the ultrawideband antenna. Chernyshev S. L., Vylenskyy A. R. Problems of diffraction and propagation of electromagnetic waves: interdepartmental collection of scientific papers. MIPT. p. 113-120.

Ymmoreev, I.Y. (2001). Radiation of ultrawideband signals. Ymmoreev Y. Y., Synyavyn A. N. Antennas. Issue. 1, p. 8-16. 
Terenov, D.B., Gutsol, T.D. (2015). Analysis of content management methods in projects. National production and economy in the conditions of reform: the state and prospects of innovation development and interregional integration, pp. 23-25

Ymmoreev, I.Y. (2010). Spatio-temporal changes UWB signals during the emission and reception of large antennas. Ymmoreev I. Y. IV All-Russian Conference "Radiolocation and Radio Communication" Institute of Radio Engineering and Electronics, Russian Academy of Sciences, 29 November -3 December. p. 615-620.

Antennas and microwave devices. (1972). Calculation and design of antenna arrays and their radiating elements. D.I. Voskresensky. Moscow: Sov. radio, 318 p.

Kalinichev, V.I. (2009). Ultra-wideband mirror antenna with horn irradiator H-shaped cross section. Kalinichev V.I., Kaloshin V.A., Pangonis L.I. III All-Russian Conference "Radiolocation and radio communication", IREE RAS. p. 68-71.

Kun Z. (1967). Microwave Antennas. Z. Kyun; translation from German under the editorship of M.P. Dolukhanov. L., Shipbuilding, 518 p.

Potapsky, P., Garasimchuk, I., Kozak, A., Mikhailova, M. (2015). Theoretical justification for creating an impulse generator for increasing the immunity of animals. MOTROL Commission of motorization and energetics in agriculture: Polish Academy of sciences. Vol. 17, №.5, p. 62-65.

Potapskyy, P., Harasymchuk, I., Pantsyr, Yu. (2015). Excitation of oscillations in a prismatic cavity by means of a rectangular waveguide. MOTROL Commission of motorization and energetics in agriculture: Polish Academy of sciences. Vol. 17, №.5. p. 66-72.

\section{BADANIA ANTENY SZEROKOPASMOWEGO PIRAMIDALNEGO TURBOWEGO PROMIENNIKA DO NISZCZENIA SZKODNIKÓW W GLEBACH}

Streszczenie. Energia elektromagnetyczna może zmieniać procesy metaboliczne i biosyntezy oraz w pewnych parametrach pulsacyjnego pola elektromagnetycznego - częstość powtórzeń impulsu, cykl pracy, moc, ekspozycję, spowolnić i hamować wzrost komórkowy. Napromieniowanie w zakresie milimetrów RNA i DNA zawierających wirus prowadzi do zmniejszenia ich zakaźności. Hamowanie wzrostu bakteryjnych kultur, zmian aktywności fagocytowej, biosyntez białka, ultrastrukturalnych zmian w komórkach pod wpływem EMF w zakresie ultra-wysokiej częstotliwości. W eksperymentach z mikroorganizmami ustalono, że biologiczny wpływ działania pól elektromagnetycznych na mikroorganizmy był rezonansowy. Jednym z głównych mechanizmów hamowania promieniowania ultra-wysokiej częstotliwości na szkodliwe mikroorganizmy jest rola błon biologicznych w reakcjach mikroorganizmów na EMF. Istotne znaczenie mają zjawiska elektryczne występujące w biomembranach.

Słowa kluczowe: Antena, elektromagnetyczna, energia, promiennik, transformacja Fouriera, transmisja fal, apertura, biotechnologia, szerokopasmowe impulsy 